\title{
Predictors of seizure outcome following resective surgery for drug-resistant epilepsy associated with focal gliosis
}

\author{
Gopal K. Dash, DM, ${ }^{1,3}$ Chaturbhuj Rathore, DM,,2 Malcolm K. Jeyaraj, DM,1,4 \\ Pandurang Wattamwar, DM, ${ }^{1,5}$ Sankara P. Sarma, PhD, ${ }^{6}$ and Kurupath Radhakrishnan, DM1,7 \\ ${ }^{1}$ R. Madhavan Nayar Center for Comprehensive Epilepsy Care, Department of Neurology, Sree Chitra Tirunal Institute for \\ Medical Sciences and Technology, Trivandrum, Kerala; ${ }^{2}$ Department of Neurology, Smt. B. K. Shah Medical Institute and \\ Research Center, Sumandeep Vidyapeeth, Vadodara, Gujarat; ${ }^{3}$ Department of Neurology, Narayana Hrudayalaya Hospital, \\ Bengaluru, Karnataka; ${ }^{4}$ Department of Neurology, Stanley Medical College, Chennai, Tamilnadu; ${ }^{5}$ Department of Neurology, \\ United CIIGMA Hospital, Aurangabad, Maharashtra; ${ }^{6}$ Achutha Menon Center for Health Science Studies, Sree Chitra Tirunal \\ Institute for Medical Sciences and Technology, Trivandrum, Kerala; and ${ }^{7}$ Amrita Advanced Epilepsy Centre, Department of \\ Neurology, Amrita Institute of Medical Sciences, Kochi, Kerala, India
}

OBJECTIVE The authors studied the clinical characteristics and postoperative outcomes of drug-resistant epilepsy associated with focal gliosis.

METHODS From their epilepsy surgery database, the authors selected the patients with drug-resistant epilepsy and MRI-defined focal gliosis who underwent focal resective surgery. All patients underwent standard presurgical evaluation. Intracranial electroencephalography (EEG) was performed in patients with discordant presurgical data, ill-defined lesions, and lesions close to eloquent regions. Completeness of resection was defined on the basis of extraoperative and intraoperative electrocorticography studies. Favorable postoperative outcome was defined as Engel class I outcome during the last 2 years of follow-up.

RESULTS Sixty-six patients fulfilled inclusion criteria. An initial precipitating injury was present in 38 (57.6\%) patients, mainly in the form of perinatal injury $(n=10)$, trauma $(n=10)$, and meningoencephalitis $(n=8)$. Gliosis involved a single lobe in $38(57.6 \%)$ patients and 2 adjacent lobes in $14(21.2 \%)$ patients; the remaining $14(21.2 \%)$ patients had multilobar gliosis. In patients with unilobar or bilobar gliosis, the posterior region of the head was involved in $34(65 \%)$ patients and the frontal lobes in $12(23 \%)$ patients. During a median follow-up of 4 years (range 2-9 years), 41 (62.1\%) patients had favorable outcome. On multivariate analysis, the presence of a well-defined aura $(p=0.019)$, electrocorticographically defined completeness of resection $(p=0.024)$, and normal postoperative EEG findings at 1 year $(p=0.003)$ were predictive of favorable postoperative seizure outcome.

CONCLUSIONS Focal gliosis is a common etiology for drug-resistant extratemporal epilepsy in developing countries and is most often located in the posterior region of the head. The majority of these patients have perinatal injuries or neurological infections as initial precipitating injuries. Patients with focal gliosis have good postoperative seizure outcomes after well-planned resective surgery.

https://thejns.org/doi/abs/10.3171/2018.3.JNS172949

KEYWORDS focal gliosis; epilepsy surgery; seizure outcome

$\mathrm{F}$ OCAL gliosis or encephalomalacia has long been recognized as a cause of drug-resistant epilepsy. ${ }^{13,22} \mathrm{Fo}-$ cal gliosis can result from perinatal strokes, perinatal hypoxia and hypoglycemia, meningoencephalitis, head trauma, and previous surgical procedures. It is one of the most common substrates for drug-resistant extratemporal lobe epilepsy in developing countries. ${ }^{89}$ In a series of 71 patients from India who underwent focal extratemporal resections for drug-resistant epilepsy, the most common etiologies were focal gliosis in 27 (38\%) patients and focal cortical dysplasia (FCD) in 19 (27\%) patients. ${ }^{9}$

Similar to patients with other types of structural lesions, patients with drug-resistant epilepsy and focal gliosis on MRI may benefit from well-planned epilepsy surgery. However, there is a relative dearth of information about the potential problems faced during the presurgical

ABBREVIATIONS AED = antiepileptic drug; ECOG = electrocorticography; EEG = electroencephalography; FCD = focal cortical dysplasia; IED = interictal discharge; VEEG = video-EEG.

SUBMITTED November 22, 2017. ACCEPTED March 6, 2018.

INCLUDE WHEN CITING Published online August 24, 2018; DOI: 10.3171/2018.3.JNS172949. 
evaluation of patients with focal gliosis, their postsurgical seizure outcome, and the predictors of postoperative outcome. As focal gliosis is a commonly encountered substrate for drug-resistant epilepsy at our center, we planned this study with the aim of studying the postoperative seizure outcome and its predictors in a group of consecutive patients who underwent focal resective surgery for drugresistant epilepsy associated with focal gliosis.

\section{Methods}

\section{Study Setting and Patient Selection}

The study was conducted at Sree Chitra Tirunal Institute for Medical Sciences and Technology, Trivandrum, Kerala, India. From a prospectively maintained database, we selected patients who underwent focal resective surgery for drug-resistant epilepsy associated with MRI-defined focal gliosis from January 2001 to December 2009 and had a minimum of 2 years of postoperative follow-up. Patients who underwent hemispherotomy were excluded from this study. We previously described our presurgical evaluation protocol, which basically consists of detailed clinical evaluation, neuropsychological evaluation, longterm video-electroencephalography (VEEG) monitoring for semiology and interictal and ictal electroencephalography (EEG) data, and high-resolution MRI., ${ }^{6,20,29,33}$ Whenever indicated, we conducted additional tests, including intracranial EEG, as part of the presurgical evaluation. ${ }^{29}$

\section{MRI Evaluation}

MRI was performed with a 1.5-T superconducting magnet (Magnetom Avanto SQ-engine, Siemens) by using a 12-channel phased-array matrix head coil. ${ }^{33}$ We used the following sequences during the routine epilepsy protocol: T2-weighted turbo spin-echo (TR/TE $3010 \mathrm{msec} / 81$ msec, number of excitations [NEX] 1), T1-weighted spinecho (TR/TE $468 \mathrm{msec} / 11 \mathrm{msec}$, NEX 1), and 2D FLAIR (TR/TE/TI $9000 \mathrm{msec} / 112 \mathrm{msec} / 2500 \mathrm{msec}$, NEX 1, slice thickness $5 \mathrm{~mm}$ ) in the axial plane using a $0.5-\mathrm{mm}$ intersection gap and 5-mm section thickness. Additionally, we acquired T2-weighted (TR/TE $5500 \mathrm{msec} / 110 \mathrm{msec}$, NEX 1) images in the sagittal plane, and T2-weighted high-resolution (TR/TE $6560 \mathrm{msec} / 109 \mathrm{msec}$, NEX 1, slice thickness $3 \mathrm{~mm}$ ), FLAIR (TR/TE/TI $9000 \mathrm{msec} / 112$ msec/2500 msec, NEX 1, slice thickness $5 \mathrm{~mm}$ ), and 3D fast low-angle shot (TR/TE $11 \mathrm{msec} / 4.94 \mathrm{msec}$, NEX 1, matrix $256 \times 256$, slice thickness $1.5 \mathrm{~mm}$ ) images in the coronal plane with the imaging plane perpendicular to the long axis of the hippocampus. Gradient echo T2*-weighted imaging (TR/TE $35 \mathrm{msec} / 10 \mathrm{msec}$, flip angle [FA] $15^{\circ}$, NEX 1, slice thickness $1.5 \mathrm{~mm}$ ) or susceptibility-weighted imaging (TR/TE $48 \mathrm{msec} / 40 \mathrm{msec}$, NEX 1, slice thickness $1.2 \mathrm{~mm}$ ) were also routinely performed. The specialized protocol included high-resolution axial T2-weighted (TR/ TE $8680 \mathrm{msec} / 104 \mathrm{msec}$, FA $90^{\circ}$, NEX 4, slice thickness $3 \mathrm{~mm}$ ) and axial proton density-weighted (TR/TE 3010 $\mathrm{msec} / 14 \mathrm{msec}$, NEX 1, slice thickness $5 \mathrm{~mm}$ ) images localized over the area of the gliosis. In addition, volumetric 3D FLAIR (TR/TE/TI $5000 \mathrm{msec} / 415 \mathrm{msec} / 1800 \mathrm{msec}$, NEX 1, slice thickness $0.9 \mathrm{~mm}$ ) images were acquired in the sagittal plane covering the entire brain from 2008 onward.
All MR images were initially viewed by one of the epilepsy neuroradiologists, and the findings were recorded in case records. For this study, we again reviewed all MR images. Based on the MRI findings, we recorded the location and extent of gliosis. The gliosis was classified as unilobar (temporal, frontal, parietal, or occipital), bilobar (involvement of 2 adjacent lobes), and multilobar.

\section{Long-Term VEEG Monitoring}

We used the standard 10-20 system of electrode placement with additional anterior temporal (T1 and T2) electrodes during long-term VEEG monitoring, as we previously described. ${ }^{6,29}$ We used sphenoidal electrodes in selected patients with temporal lobe epilepsy and discordant or nonlocalizing/nonlateralizing interictal and ictal data. We used standard longitudinal bipolar and common average montages while reviewing the EEG records. The interictal and ictal data were reviewed by 2 of the epileptologists involved in the epilepsy program during the morning VEEG meetings. The interictal discharges (IEDs) confined to anterior and midtemporal electrodes (F7, F8, $\mathrm{T} 1, \mathrm{~T} 2, \mathrm{~T} 3$, and T4) were defined as temporal IEDs, while others were classified as extratemporal IEDs. In those patients with a unilateral MRI abnormality and concordant IEDs, we recorded minimum of 2 seizures; a minimum 5 seizures were recorded in patients with discordant interictal or ictal data. The ictal onset rhythms were classified as focal, regional, hemispheric, or diffuse. We defined focal ictal onset as the presence of a well-defined ictal rhythm over the same lobe as that of gliosis. When ictal onset was noted simultaneously over 2 or more adjacent lobes close to the lesion, it was defined as regional ictal onset. Other ictal rhythms were classified as lateralizing (on the side of lesional hemisphere) or diffuse. Similarly, the interictal discharges were classified as focal, regional, multifocal, and generalized. ${ }^{26}$

\section{Invasive EEG Studies}

After the initial baseline noninvasive evaluation, further decisions were made in the multidisciplinary patient management meetings. As all patients had MRI-defined lesions, further noninvasive functional studies, including SPECT and FDG-PET, were not undertaken, mostly to avoid the additional financial burden to the patients. All patients with well-defined gliotic lesions over the noneloquent areas and concordant VEEG data were directly selected for surgery. Patients with discordant MRI and EEG data, ill-defined MRI lesions, or gliosis at or near the eloquent areas were planned for intracranial EEG and cortical stimulation. We used 4-8 contact subdural strip electrodes, 4-8 contact depth electrodes, and 16-64 contact subdural grids for intracranial EEG. Intracranial ictal onset was defined as focal (when $\leq 3$ adjacent electrodes were involved at onset), regional (involvement of 4-8 adjacent electrodes), and diffuse (when $>8$ contacts were involved simultaneously at onset). Extraoperative stimulation studies were performed to map eloquent cortices according to the previously described protocol. ${ }^{5}$ Preresection intraoperative electrocorticography (ECoG) was performed in patients who underwent direct surgery with- 
out intracranial EEG using grid and strip electrodes, and postresection ECoG was performed in all patients. The intraoperative ECoG studies were tailored to define the extent of the irritative zone (i.e., areas showing interictal spikes) in relation to the lesion.

During surgery, the extent of resection was primarily defined by the MRI-identified abnormality, intraoperative findings, and ECoG findings. Resections were tailored to include the areas of persistent spikes with a frequency of $\geq 1 \mathrm{~Hz}$, rhythmic spikes, bursts of paroxysmal fast activity, or recruiting rhythms on ECoG, if this could be achieved safely without affecting the eloquent regions. We have defined the completeness of resection on the basis of extraoperative and intraoperative ECoG findings. ${ }^{28}$ For those patients who underwent extraoperative $\mathrm{ECoG}$, completeness of resection was defined as complete removal of areas showing ictal onset, early seizure propagation, paroxysmal fast activity, and rhythmic spiking of $\geq 1 \mathrm{~Hz}$. For those patients who underwent intraoperative ECoG, completeness of resection was defined as complete removal of areas showing paroxysmal fast activity and rhythmic spikes along with more than $75 \%$ reduction in the number of random spikes on postoperative ECoG. ${ }^{28}$

\section{Outcome Assessment}

Postoperatively, all patients were evaluated in person by one of the epileptologists at 3 months, 1 year, and subsequently at yearly intervals until 5 years. Seizure recurrence, if any, and seizure frequency were noted during personal interviews. After 5 years, patients who remained seizure free and were unable to visit the clinic yearly were followed up through postal interview. In case of any seizure recurrence, patients were asked to contact us in person or by telephone and were asked to maintain seizure diaries as a standard practice. These data were entered into the prospectively maintained database. For this study, we classified the postoperative seizure outcome according to the Engel epilepsy surgery outcome classification. ${ }^{12} \mathrm{~Pa}-$ tients in Engel class I during the last 2 years of follow-up were classified as having a favorable outcome, while other patients were classified as having an unfavorable outcome. A 40-minute scalp EEG was done for all patients at each follow-up visit for 3 years. ${ }^{32}$ Due to the financial constraints, postoperative MRI was not done routinely and was undertaken selectively in patients with unfavorable outcomes. If patients remained seizure free, gradual antiepileptic drug (AED) withdrawal was started at 3 months in patients who were taking 2 or more antiepileptic drugs, while withdrawal was started at 1 year in those patients who were on monotherapy. AEDs were withdrawn slowly over 2-3 years in a sequential predefined manner as described previously. ${ }^{24,30}$ Patients who had seizure recurrence during AED withdrawal or those who had persistent seizures following surgery were managed on an individual basis.

\section{Standard Procedures}

All patients or caregivers gave written informed consent for undergoing treatment. As this study is a retrospective audit of the standard patient management protocols,
TABLE 1. Clinical characteristics of patients with focal gliosis

\begin{tabular}{lc}
\hline \multicolumn{1}{c}{ Characteristic } & No. of Patients $(\%)$ \\
\hline Antecedent events & $38(57.6)$ \\
Perinatal insults & $10(26.3)$ \\
Trauma & $10(26.3)$ \\
Febrile seizures & $9(23.7)$ \\
Stroke & $1(2.6)$ \\
Meningoencephalitis & $8(21.1)$ \\
\hline Seizure types & \\
Aura & $34(51.5)$ \\
Complex partial seizures & $65(98.5)$ \\
Focal to bilat TCS & $46(69.7)$ \\
Status epilepticus & $7(10.6)$ \\
Preop neurological deficits & \\
Hemianopia & $32(49)$ \\
Hemiparesis & $13(19.7)$ \\
Sensory impairment & $2(3)$ \\
Gliosis distribution & \\
Unilobar & $38(57.6)$ \\
Bilobar & $14(21.2)$ \\
Multilobar & $14(21.2)$ \\
Postop deficits & \\
Hemianopia & $5(7.6)$ \\
Worsening of motor deficit & $1(1.5)$ \\
Aphasia & $1(1.5)$ \\
Sensory impairment & $1(1.5)$ \\
\hline
\end{tabular}

TCS = tonic-clonic seizures.

the institutional ethics committee waived the individual patient consent. All necessary clearances for publication were obtained from the research and publication cell and the director of the institution.

\section{Statistical Analysis}

We used descriptive statistics to summarize the demographic and clinical data. We compared different preoperative and postoperative variables between favorable and unfavorable outcome groups by using Pearson's chisquare test and Fisher's exact test, where appropriate. We used time-to-event (from surgery to seizure recurrence) Kaplan-Meier survival curves to study the seizure outcome. A p value of $<0.05$ was considered as significant. All analyses were performed using IBM SPSS Statistics for Windows (version 21.0, IBM Corp.).

\section{Results \\ Clinical Characteristics}

During the study period, 66 patients underwent focal resections for drug-resistant epilepsy due to focal gliosis. This accounted for $8.5 \%$ of all epilepsy surgeries $(\mathrm{n}=774)$ and $46 \%$ of all extratemporal resections $(\mathrm{n}=$ 143) during the study period. The clinical characteristics of these patients are summarized in Table 1. Characteristics of patients with favorable and unfavorable seizure outcomes are compared in Tables 2 and 3. There were 49 males and 17 females. The mean age at the time of surgery was $19.8 \pm 10.1$ years (median 17 years, range 5-40 years), the mean age at seizure onset was $8.3 \pm 6.1$ years 
TABLE 2. Predictors of seizure outcome following resective surgery for focal gliosis on univariate analysis

\begin{tabular}{lccc}
\hline \multicolumn{1}{c}{ Parameter } & $\begin{array}{c}\text { Favorable } \\
\text { Outcome } \\
(\mathrm{n}=41)^{*}\end{array}$ & $\begin{array}{c}\text { Unfavorable } \\
\text { Outcome } \\
(\mathrm{n}=25)\end{array}$ & $\begin{array}{c}\mathrm{p} \\
\text { Value }\end{array}$ \\
\hline Presence of antecedents, $\mathrm{n}(\%)$ & $22(53.7)$ & $16(64)$ & 0.410 \\
\hline Mean age at surgery in yrs ( \pm SD) & $18.66 \pm 7.4$ & $21.6 \pm 9.1$ & 0.127 \\
\hline $\begin{array}{l}\text { Mean duration of epilepsy in yrs } \\
\quad \pm \text { SD) }\end{array}$ & $11.9 \pm 6.7$ & $13.1 \pm 7.8$ & 0.528 \\
\hline SGTCS, $\mathrm{n}(\%)$ & $27(65.8)$ & $19(76.0)$ & 0.384 \\
\hline Status epilepticus, $\mathrm{n}(\%)$ & $5(12.2)$ & $2(8.0)$ & 0.701 \\
\hline Presence of aura, $\mathrm{n}(\%)$ & $26(63.4)$ & $8(32.0)$ & 0.013 \\
\hline Preoperative FND, $\mathrm{n}(\%)$ & $26(63.4)$ & $12(48.0)$ & 0.219 \\
\hline Unilobar gliosis, $\mathrm{n}(\%)$ & $23(56.1)$ & $18(72.0)$ & 0.802 \\
\hline Focal/regional IEDs in EEG, $\mathrm{n}(\%)$ & $23(56.1)$ & $8(32.0)$ & 0.060 \\
\hline Focal/regional ictal onset, $\mathrm{n}(\%)$ & $26(63.4)$ & $17(68.0)$ & 0.705 \\
\hline Beta onset on scalp EEG, $\mathrm{n}(\%)$ & $16(39.0)$ & $10(40.0)$ & 0.937 \\
\hline Multilobar resection, $\mathrm{n}(\%)$ & $5(12.2)$ & $4(16.0)$ & 0.720 \\
\hline Completeness of resection, $\mathrm{n}(\%) \dagger$ & $39(95.1)$ & $17(68)$ & 0.005 \\
\hline Normal postop EEG, $\mathrm{n}(\%)$ & & & \\
7 days & $27(65.9)$ & $9(36.0)$ & 0.018 \\
3 mos & $27(65.9)$ & $9(36.0)$ & 0.018 \\
1 yr & $32(78.0)$ & $9(36.0)$ & 0.001 \\
\hline
\end{tabular}

SGTCS = secondarily generalized tonic-clonic seizure.

* Defined as Engel class I outcome.

$\dagger$ Completeness as defined by ECoG.

(median 6 years, range 0.1-21 years), and the mean duration of epilepsy was $13.3 \pm 9$ years (median 10 years, range $1-32$ years). An initial precipitating injury was noted in 38 (57.6\%) patients, which mainly included perinatal injury in 10 patients, trauma in another 10 patients, and meningoencephalitis in 8 patients. Thirty-four patients $(51.5 \%)$ reported well-defined auras. Complex partial seizures were noted in $65(98.5 \%)$ patients, while $46(69.7 \%)$ patients had additional secondary generalized seizures. Forty-seven (71\%) patients had preoperative focal deficits, which included hemianopia in $32(49 \%)$ patients, mild hemiparesis in $13(20 \%)$ patients, and cortical sensory impairment in $2(3 \%)$ patients.

\section{Distribution of Gliosis on MRI}

Gliosis was restricted to the single lobe in 38 (57.6\%) patients, while $14(21.2 \%)$ patients had involvement of 2 adjacent lobes and 14 (21.2\%) patients had multilobar gliosis. In the unilobar group, gliosis involved the occipital lobe in 17 patients, frontal lobe in 12 patients, parietal lobe in 5 patients, and temporal lobe in 4 patients. The commonly involved 2 adjacent lobes were parietal and occipital lobes in 8 patients, occipital and temporal lobes in 4 patients, and frontal and temporal lobes in 2 patients. Thus, gliosis was located over the posterior region of the head in 34 (65\%) patients with unilobar or bilobar involvement. Various types of gliosis in this series are presented in Fig. 1.

\section{EEG Data}

Twenty-one (31.8\%) patients required invasive monitoring before resective surgery. Patients were monitored for 1-6 days during scalp VEEG and for 5-13 days during intracranial EEG. During scalp VEEG, the median number of recorded seizures was 3 per patient (range 2-15) while it was 8 per patient (range 3-21) during intracranial EEG. The scalp-recorded ictal onset was focal or regional in $43(65.1 \%)$ patients and hemispheric or diffuse in 23 (34.9\%) patients. The pattern of ictal onset on scalp EEG was in the form of low-voltage beta activity in 26 (39.4\%) patients, either alpha or theta activity in $15(22.7 \%)$ patients, and slower activity in $25(37.9 \%)$ patients. Interictal discharges were focal or regional in 31 (47.0\%) patients.

\section{Surgical Procedures}

Lesionectomy was performed in 51 (77.3\%) patients, lobectomy in $6(9.1 \%)$ patients, and multilobar resection in $9(13.6 \%)$ patients. Perioperatively, the resection was considered incomplete due to the persistent spikes on postoperative ECoG in $10(15.2 \%)$ patients, as these patients had resection margins close to eloquent regions. The remaining $56(84.8 \%)$ patients had more than a $75 \%$ reduction in spikes on postoperative ECoG, and these resections were considered to have been complete. MRI characteristics and surgical resections in 3 of the patients are illustrated in Figs. 2-4.

\section{Pathology}

We reviewed the histopathological reports of all the cases. Histopathological examination of the lesions showed neuronal loss, astrogliosis, and microglia formation without any evidence of inflammation or neoplasm in all the cases. This was reported as nonspecific gliosis. However, the presence of dysplasia was not specifically reported, and hence we cannot comment on this feature.

\section{Postoperative Outcome}

During a median follow-up of 4 years (range 2-9 years), $41(62.1 \%)$ patients had Engel class I outcome, 11 (16.7\%)

TABLE 3. Predictors of seizure outcome after resective surgery for focal gliosis on multivariate analysis

\begin{tabular}{lcccccc}
\hline \multicolumn{1}{c}{ Variable } & Regression Coefficient & SE & Wald Statistic & DF & p Value & Adjusted OR (95\% Cl) \\
\hline Presence of aura & -1.532 & 0.652 & 5.512 & 1 & 0.019 & $0.22(0.06-0.78)$ \\
\hline Completeness of resection & 2.198 & 0.973 & 5.105 & 1 & 0.024 & $9.0(1.34-60.65)$ \\
\hline IEDs on 1 yr postop EEG & 1.940 & 0.651 & 8.876 & 1 & 0.003 & $6.97(1.94-24.95)$ \\
\hline
\end{tabular}

$\mathrm{DF}=$ degree of freedom; $\mathrm{SE}=$ standard error

${ }^{*}$ Completeness as defined by ECoG. 
Dash et al.
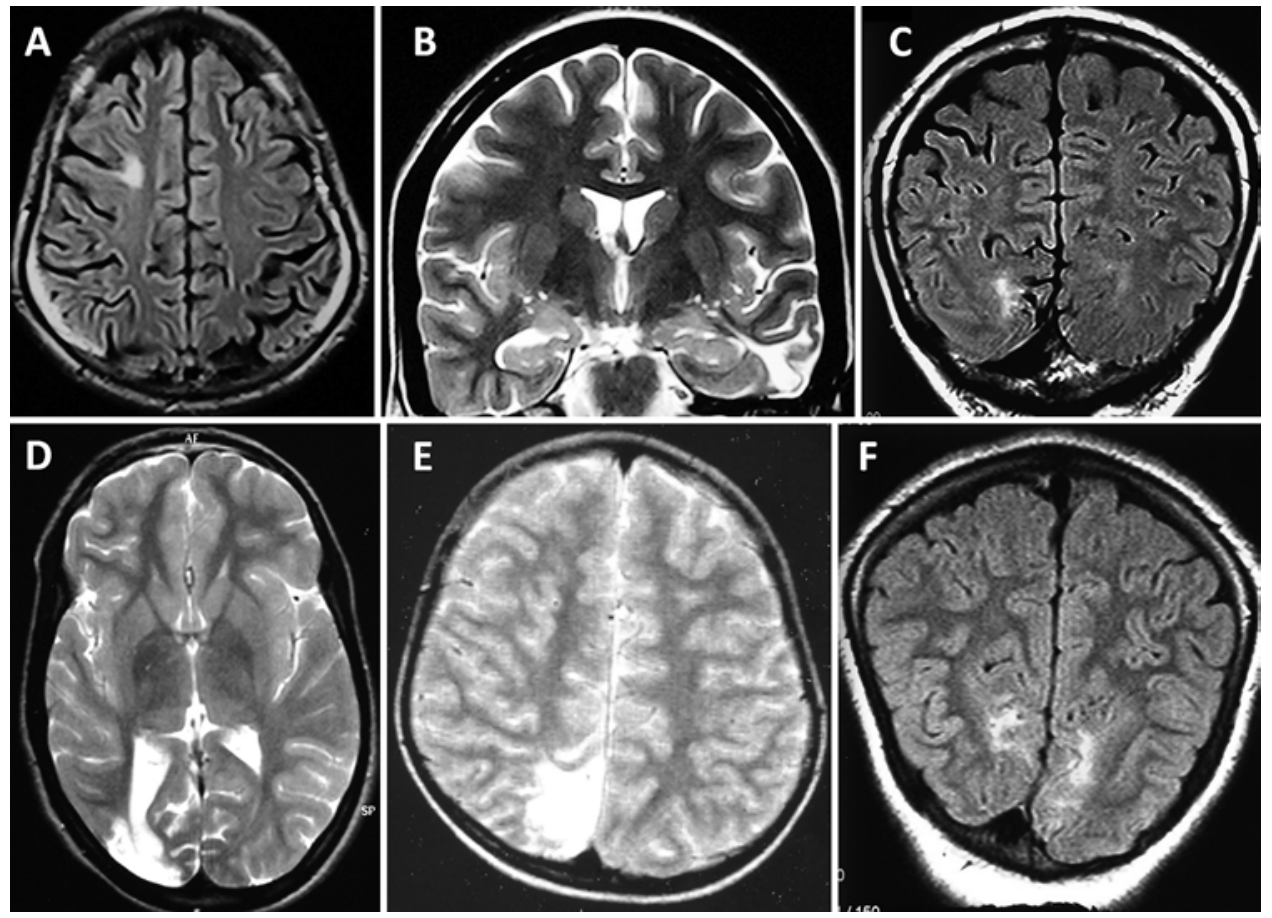

FIG. 1. MR images showing the different forms of gliosis that were surgically treated during the study period. A: Right frontal gliosis related to neurocysticercal lesion. B: Left temporal gliosis of unknown etiology. C: Right occipital gliosis probably related to perinatal hypoxic-ischemic injury. D: Right lateral occipital gliosis probably related to perinatal hypoxic-ischemic injury. E: Right parietal gliosis related to perinatal injury. F: Bilateral asymmetrical occipital gliosis due to perinatal hypoxic injury. All electroclinical data suggested left occipital seizure origin in this patient. The patient is seizure free following a left occipital lobectomy.

had class II outcome, 2 (3.0\%) had class III outcome, and $12(18.2 \%)$ patients had class IV outcome. Actuarial analysis showed that 14 of 25 patients (56\%) in the unfavorable outcome group had recurrences within the first 3 months of surgery (Fig. 5). Overall, 19 (76\%) patients had seizure recurrence within the 1st year after surgery. Of these 19 patients, only $3(15.8 \%)$ patients subsequently became seizure free at the last follow-up. In the favorable outcome
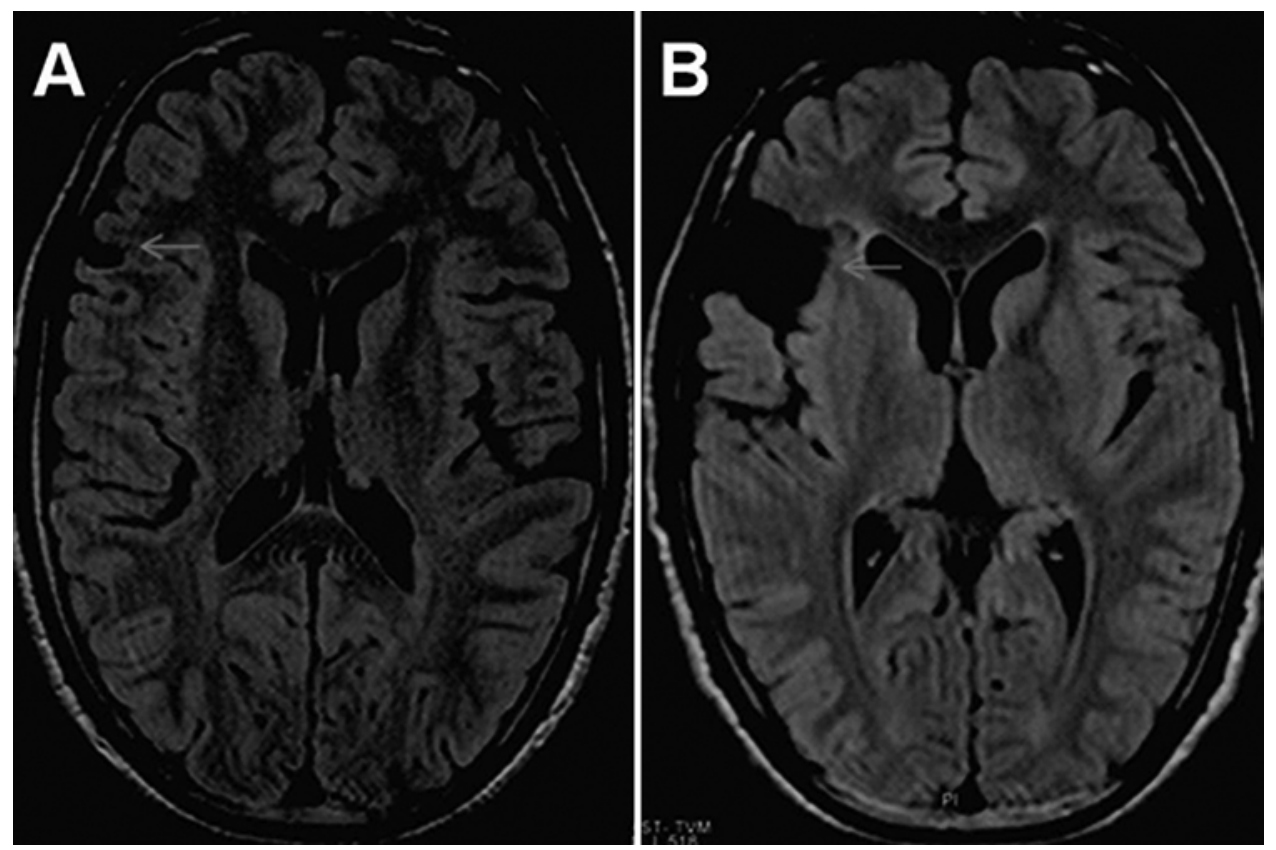

FIG. 2. Axial FLAIR MRI sequences obtained in a 20-year-old man with drug-resistant epilepsy due to posttraumatic right frontal gliosis (arrow) (A). The patient is seizure free at 5 years' follow-up after lesionectomy (arrow) (B). 


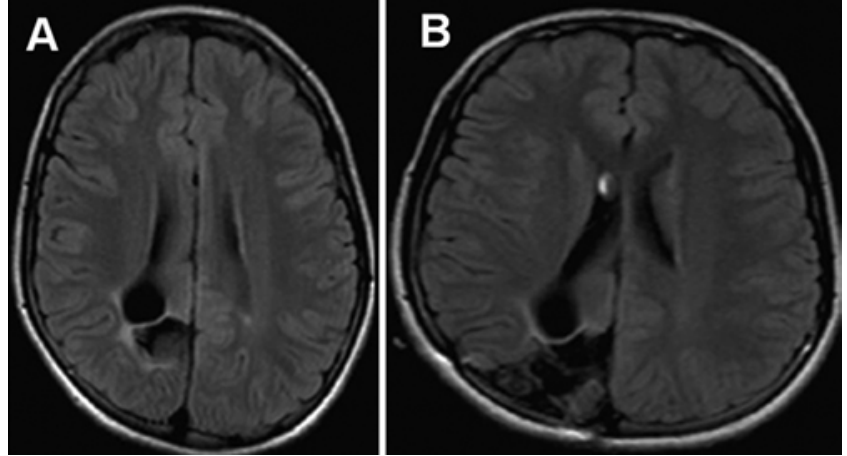

FIG. 3. Axial FLAIR MRI sequences obtained in a 14-year-old boy with drug-resistant epilepsy and right parietooccipital gliosis related to perinatal hypoxia (A). The patient is seizure free at 4 years after lesionectomy (B).

group, AEDs were discontinued in 10 (24.4\%) patients, while doses of AED were reduced in another 30 (45.5\%) patients. Ten patients with late recurrences had recurrences related to AED withdrawal and only one of them became seizure free at the last follow-up.

\section{Predictors of Postoperative Seizure Outcome}

The characteristics of patients with favorable and unfavorable seizure outcomes are compared in Tables 2 and 3 . The presence of well-defined aura $(63.4 \%$ vs $32.0 \%$, p $=0.013)$ and completeness of resection $(95.1 \%$ vs $68 \%, \mathrm{p}$ $=0.005$ ) were significantly associated with favorable outcome. Presence of IEDs on the postoperative EEG on the 7 th day, 3 months, and at 1 year predicted unfavorable outcome. On multivariate analysis, the presence of a welldefined aura $(\mathrm{p}=0.019)$, completeness of resection $(\mathrm{p}=$ $0.024)$, and normal postoperative EEG results at 1 year ( $p$ $=0.003$ ) were predictive of favorable postoperative seizure outcome.

\section{Postoperative Complications}

One patient, who underwent posterior quadrantic resection, died of sepsis and acute respiratory distress syndrome 2 weeks after surgery. Five patients developed new hemianopia. Three patients developed transient deficits, in the form of worsening of motor deficit, new sensory deficit, and aphasia. All transient deficits improved in the first postoperative month.

\section{Discussion}

Focal gliosis is a common substrate for drug-resistant epilepsy, particularly extratemporal lobe epilepsy, in India, as shown in this study and in a previous study from our center. ${ }^{9}$ This is in contrast to the reports from developed countries, where FCD is the most commonly reported cause of drug-resistant extratemporal lobe epilepsy. ${ }^{10,14,21}$ In a large series of 165 patients from Germany who underwent focal extratemporal resections for drug-resistant epilepsy, both FCD and neoplastic lesions were noted in $34.4 \%$ patients each, while only $12.3 \%$ patients had gliosis..$^{10}$ The major causes for gliosis, apart from the trauma, are various forms of hypoxic and vascular perinatal in-
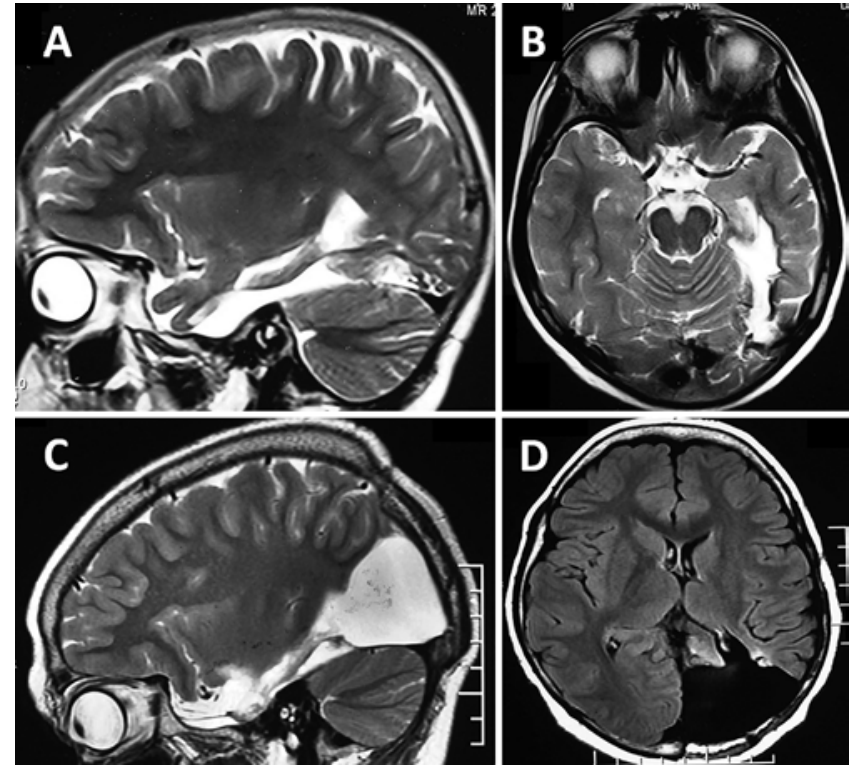

FIG. 4. A and B: Preoperative MR images obtained in a 7-year-old boy with drug-resistant epilepsy and left temporooccipital gliosis, possibly related to perinatal injury. $\mathbf{C}$ and $\mathbf{D}$ : The patient has been seizure free for the last 8 years after lesionectomy.

juries and neurological infections, both of which were noted in half of our patients who had a history of antecedent events. Both of these factors are more commonly encountered in developing countries due to the less developed perinatal care facilities and a high incidence of communicable diseases. Perinatal problems and difficult labor have been documented as important risk factors for epilepsy in a population-based study from our center. ${ }^{16}$ Perinatal injuries, both hypoxia and hypoglycemia, were also found to be the most common underlying etiology in $50 \%$ of pediatric patients from India with drug-resistant epilepsy. ${ }^{36}$ This is further illustrated by the fact that the majority of our patients had gliosis located over posterior regions of the head, which is a common site of involvement in perinatal hypoxic-ischemic injuries and perinatal hypoglycemia. . $^{3,25,27}$ Thus a higher incidence of perinatal injuries contributing to parieto-occipital gliosis is a major reason for a greater proportion of patients with focal gliosis and drug-resistant extratemporal lobe epilepsy in developing countries.

Our results show that resective epilepsy surgery is quite effective and safe in patients with focal gliosis. Approximately $62 \%$ of patients in our series became seizure free postoperatively. This is similar to the seizure outcomes reported after various extratemporal focal resections. ${ }^{10,21,35}$ Only a few other studies, all with a small number of patients, have specifically reported seizure outcome after focal resection for gliosis. . $, 7,17,22$ In a series of 21 patients from India who underwent focal resection for encephalomalacia, 17 (81\%) had Engel class I outcome at a median postoperative follow-up of 21 months. ${ }^{4}$ In another series of 17 patients with frontal gliosis, $12(70 \%)$ had favorable seizure outcome after focal resections. ${ }^{17}$ Recently, ulegyria, a form of gliosis, has also been reported to be associated with drug-resistant epilepsy. ${ }^{19,37}$ Focal gliosis has also 


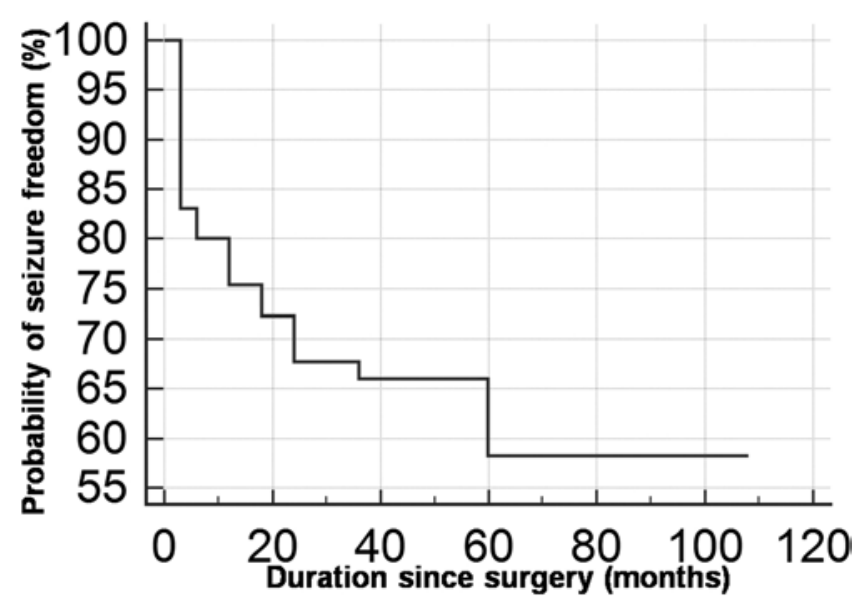

FIG. 5. Kaplan-Meier survival curve for seizure freedom following surgery.

been observed as one of the etiologies in studies that have reported seizure outcomes following various extratemporal resections. In studies reporting the outcome following posterior cortex resection, the proportion of patients with focal gliosis has varied from $12 \%$ to $35 \%$, with the greatest proportion from a study in Brazil. ${ }^{8,11,15}$ Thus, the present study represents one of the largest series of patients with focal gliosis who underwent resection for drug-resistant epilepsy.

Our results also show that the majority of these patients with unfavorable outcome have early seizure recurrence, within the 1st year following surgery. Most of these patients subsequently continue to have seizures during long-term follow-up. This is in contrast to temporal lobe epilepsy surgeries where many patients with early recurrence have seizure remission on long-term follow-up. ${ }^{1} \mathrm{On}$ the other hand, the majority of late recurrences in these patients are related to AED withdrawal. These results are similar to those in our previous study of AED withdrawal in extratemporal resections, indicating that AEDs should be carefully withdrawn in these patients. ${ }^{24}$

We found that the presence of a well-defined aura and the completeness of resection as defined on the basis of intraoperative and extraoperative ECoG were predictive of favorable postoperative seizure outcome. The presence of a well-defined aura has been shown to predict favorable seizure outcome in previous studies. ${ }^{11,34}$ It helps in better localization of the epileptogenic zone and may indicate relatively restricted initial ictal activation. Absence of auras, on the other hand, may indicate more widespread epileptogenic zones or more rapid seizure spread to other brain regions. The presence of frequent spikes on postresection ECoG may indicate incomplete resection of the epileptogenic zone and has been shown to predict unfavorable outcome after extratemporal resections. ${ }^{28}$ Due to the financial constraints, we could not perform postoperative MRI in all patients to confirm the completeness of resection. The presence of spikes on 1-year postoperative EEG was also predictive of unfavorable seizure outcome, as has been documented in other extratemporal resections. ${ }^{31}$

Presurgical evaluation and epilepsy surgery in patients with focal gliosis present certain specific problems. Gliosis is predominantly located in the extratemporal region and is often near eloquent areas. Gliosis resulting from perinatal injuries usually involves posterior watershed regions, close to sensory and motor cortices. ${ }^{25,27}$ Perinatal hypoxicischemic injuries and hypoglycemia usually result in bilateral parietooccipital gliosis. Often, these patients have bilateral symmetrical gliosis with significant visual impairment, precluding any focal resective surgery. Patients with predominantly unilateral gliosis also have minor contralateral structural changes, which make presurgical evaluation more complex. The MRI-defined areas of gliosis often have ill-defined margins, causing difficulty in delineating the exact extent of the structural abnormality and the epileptogenic zone, often requiring intracranial EEG. Thus, gliosis represents a unique surgical entity, similar to FCD and distinct from other well-defined lesions, such as low-grade neoplasms and vascular malformations. Despite these difficulties, as our results indicate, the majority of these patients can have good outcomes after a wellplanned presurgical evaluation and surgical strategy. The surgical strategy should be based on the complete resection of an MRI-defined lesion guided by anatomical landmarks, perioperative findings, and intraoperative ECoG.

The most common location for gliosis in our series was the parietooccipital region, a common site of involvement in perinatal hypoxic-ischemic injuries. ${ }^{3,25,27}$ In a postmortem series of 36 patients with hypoxic-ischemic injuries of various severities who died of unrelated reasons, it was demonstrated that the cortex undergoes continuous structural and functional changes, leading to the development of acquired dysplasia after injury. ${ }^{23}$ The presence of dysplastic tissue has also been well documented in the brain surrounding the gliosis, which has been classified as FCD type IIId. ${ }^{2}$ Perinatal injury has also been documented as one of the important risk factors in patients with histopathologically proven type I FCD. ${ }^{18}$ We can hypothesize that the presence of acquired type I FCD near glial scarring after prenatal or perinatal hypoxic-ischemic injury contributes to the epileptogenicity of gliotic tissue and the surrounding neurons. Thus, it is possible that an early injury to the vulnerable neurons in the perinatal period may result in the formation of acquired dysplasia alone or focal gliosis with dysplasia, depending on the severity of the initial injury. However, in this study, we could not specifically evaluate the presence of dysplasia surrounding the gliosis on pathology specimens, and this area requires further study.

Because this was a retrospective study, our data have certain limitations. Although we tried to include all patients with gliosis who underwent surgery during the study period, it is possible that we might have missed a small proportion of cases due to the lack of records. We could not verify the completeness of resection of structural lesions, as postoperative MR images were not available in the majority of patients. Approximately $40 \%$ of our patients had no history of antecedent events. In all probability, these patients had minor perinatal injuries, which could not be recalled or noted in the records. Lastly, as noted earlier, we could not verify the pathological findings, especially the presence of perilesional dysplasia. In spite of these 
limitations, our data provide useful information about the potential problems encountered in the presurgical evaluation of patients with this unique substrate of drug-resistant epilepsy and indicate that the majority of these patients can have favorable outcomes with a well-planned surgical strategy.

\section{Conclusions}

Our results show that focal gliosis is a common etiology for drug-resistant epilepsy, especially extratemporal epilepsy, in developing countries and is mainly located in the posterior regions in the head. The majority of these patients had perinatal injuries or meningoencephalitis as initial precipitating injuries. Our patients with focal gliosis had good postoperative seizure outcomes after wellplanned epilepsy surgery.

\section{References}

1. Berkovic SF, McIntosh AM, Kalnins RM, Jackson GD, Fabinyi GC, Brazenor GA, et al: Preoperative MRI predicts outcome of temporal lobectomy: an actuarial analysis. Neurology 45:1358-1363, 1995

2. Blümcke I, Thom M, Aronica E, Armstrong DD, Vinters HV, Palmini A, et al: The clinicopathologic spectrum of focal cortical dysplasias: a consensus classification proposed by an ad hoc Task Force of the ILAE Diagnostic Methods Commission. Epilepsia 52:158-174, 2011

3. Caraballo RH, Sakr D, Mozzi M, Guerrero A, Adi JN, Cersósimo RO, et al: Symptomatic occipital lobe epilepsy following neonatal hypoglycemia. Pediatr Neurol 31:24-29, 2004

4. Chandra PS, Ansari A, Chauhan A, Kumar A, Garg A, Sarkar C, et al: Surgery for encephalomalacias presenting with intractable epilepsy. Indian J Neurosurg 1:33-37, 2012

5. Chaudhry N, Radhakrishnan A, Abraham M, Kesavadas C, Radhakrishnan VV, Sankara Sarma P, et al: Selection of ideal candidates for extratemporal resective epilepsy surgery in a country with limited resources. Epileptic Disord 12:38-47, 2010

6. Chemmanam T, Radhakrishnan A, Sarma SP, Radhakrishnan $\mathrm{K}$ : A prospective study on the cost-effective utilization of long-term inpatient video-EEG monitoring in a developing country. J Clin Neurophysiol 26:123-128, 2009

7. Cukiert A, Olivier A, Andermann F: Post-traumatic frontal lobe epilepsy with structural changes: excellent results after cortical resection. Can J Neurol Sci 23:114-117, 1996

8. Dalmagro CL, Bianchin MM, Velasco TR, Alexandre V Jr, Walz R, Terra-Bustamante VC, et al: Clinical features of patients with posterior cortex epilepsies and predictors of surgical outcome. Epilepsia 46:1442-1449, 2005

9. Dash GK, Radhakrishnan A, Kesavadas C, Abraham M, Sarma PS, Radhakrishnan K: An audit of the presurgical evaluation and patient selection for extratemporal resective epilepsy surgery in a resource-poor country. Seizure 21:361-366, 2012

10. Elsharkawy AE, Behne F, Oppel F, Pannek H, Schulz R, Hoppe M, et al: Long-term outcome of extratemporal epilepsy surgery among 154 adult patients. J Neurosurg 108:676686, 2008

11. Elsharkawy AE, El-Ghandour NM, Oppel F, Pannek H, Schulz R, Hoppe M, et al: Long-term outcome of lesional posterior cortical epilepsy surgery in adults. J Neurol Neurosurg Psychiatry 80:773-780, 2009

12. Engel J Jr, Van Ness PC, Rasmussen TB, Ojemann LM: Outcome with respect to epileptic seizures, in Engel J Jr (ed):
Surgical Treatment of the Epilepsies, ed 2. New York: Raven Press, 1993, pp 609-621

13. Foerster O, Penfield W: The structural basis of traumatic epilepsy and results of radical operation. Brain 53:99-119, 1930

14. Jeha LE, Najm I, Bingaman W, Dinner D, Widdess-Walsh P, Lüders H: Surgical outcome and prognostic factors of frontal lobe epilepsy surgery. Brain 130:574-584, 2007

15. Jehi LE, O’Dwyer R, Najm I, Alexopoulos A, Bingaman W: A longitudinal study of surgical outcome and its determinants following posterior cortex epilepsy surgery. Epilepsia 50:2040-2052, 2009

16. Kannoth S, Unnikrishnan JP, Santhosh Kumar T, Sankara Sarma P, Radhakrishnan K: Risk factors for epilepsy: a population-based case-control study in Kerala, southern India. Epilepsy Behav 16:58-63, 2009

17. Kazemi NJ, So EL, Mosewich RK, O’Brien TJ, Cascino GD, Trenerry MR, et al: Resection of frontal encephalomalacias for intractable epilepsy: outcome and prognostic factors. Epilepsia 38:670-677, 1997

18. Krsek P, Jahodova A, Maton B, Jayakar P, Dean P, Korman $\mathrm{B}$, et al: Low-grade focal cortical dysplasia is associated with prenatal and perinatal brain injury. Epilepsia 51:2440-2448, 2010

19. Kuchukhidze G, Unterberger I, Dobesberger J, Embacher N, Walser G, Haberlandt E, et al: Electroclinical and imaging findings in ulegyria and epilepsy: a study on 25 patients. J Neurol Neurosurg Psychiatry 79:547-552, 2008

20. Lachhwani DK, Radhakrishnan K: Epilepsy surgery in India, in Lüders HO (ed): Textbook of Epilepsy Surgery. Boca Raton, FL: Taylor \& Francis, 2008, pp 134-144

21. Lee SK, Lee SY, Kim KK, Hong KS, Lee DS, Chung CK: Surgical outcome and prognostic factors of cryptogenic neocortical epilepsy. Ann Neurol 58:525-532, 2005

22. Li L: [Surgical treatment of traumatic epilepsy.] Zhonghua Wai Ke Za Zhi 29:242-243, 271, 1991 (Chinese)

23. Marín-Padilla M: Neuropathologic correlates of perinatal asphyxia. Int Pediatr 15:221-228, 2000

24. Menon R, Rathore C, Sarma SP, Radhakrishnan K: Feasibility of antiepileptic drug withdrawal following extratemporal resective epilepsy surgery. Neurology 79:770-776, 2012

25. Nikas I, Dermentzoglou V, Theofanopoulou M, Theodoropoulos V: Parasagittal lesions and ulegyria in hypoxic-ischemic encephalopathy: neuroimaging findings and review of the pathogenesis. J Child Neurol 23:51-58, 2008

26. Noachtar S, Binnie C, Ebersole J, Mauguière F, Sakamoto A, Westmoreland B: A glossary of terms most commonly used by clinical electroencephalographers and proposal for the report form for the EEG findings. Electroencephalogr Clin Neurophysiol Suppl 52:21-41, 1999

27. Oguni H, Sugama M, Osawa M: Symptomatic parieto-occipital epilepsy as sequela of perinatal asphyxia. Pediatr Neurol 38:345-352, 2008

28. Palmini A, Gambardella A, Andermann F, Dubeau F, da Costa JC, Olivier A, et al: Intrinsic epileptogenicity of human dysplastic cortex as suggested by corticography and surgical results. Ann Neurol 37:476-487, 1995

29. Rathore C, Kesavadas C, Ajith J, Sasikala A, Sarma SP, Radhakrishnan K: Cost-effective utilization of single photon emission computed tomography (SPECT) in decision making for epilepsy surgery. Seizure 20:107-114, 2011

30. Rathore C, Panda S, Sarma PS, Radhakrishnan K: How safe is it to withdraw antiepileptic drugs following successful surgery for mesial temporal lobe epilepsy? Epilepsia 52:627635,2011

31. Rathore C, Radhakrishnan K: Prognostic significance of interictal epileptiform discharges after epilepsy surgery. J Clin Neurophysiol 27:255-262, 2010

32. Rathore C, Sarma SP, Radhakrishnan K: Prognostic impor- 
tance of serial postoperative EEGs after anterior temporal lobectomy. Neurology 76:1925-1931, 2011

33. Saini J, Singh A, Kesavadas C, Thomas B, Rathore C, Bahuleyan B, et al: Role of three-dimensional fluid-attenuated inversion recovery (3D FLAIR) and proton density magnetic resonance imaging for the detection and evaluation of lesion extent of focal cortical dysplasia in patients with refractory epilepsy. Acta Radiol 51:218-225, 2010

34. Sperling MR, O'Connor MJ: Auras and subclinical seizures: characteristics and prognostic significance. Ann Neurol 28:320-328, 1990

35. Téllez-Zenteno JF, Hernández Ronquillo L, Moien-Afshari F, Wiebe S: Surgical outcomes in lesional and non-lesional epilepsy: a systematic review and meta-analysis. Epilepsy Res 89:310-318, 2010

36. Udani V: Pediatric epilepsy-an Indian perspective. Indian J Pediatr 72:309-313, 2005

37. Usui N, Mihara T, Baba K, Matsuda K, Tottori T, Umeoka S, et al: Posterior cortex epilepsy secondary to ulegyria: is it a surgically remediable syndrome? Epilepsia 49:1998-2007, 2008

\section{Disclosures}

The authors report no conflict of interest concerning the materi- als or methods used in this study or the findings specified in this paper.

\section{Author Contributions}

Conception and design: Rathore, Radhakrishnan. Acquisition of data: Dash, Jeyaraj, Wattamwar. Analysis and interpretation of data: Rathore, Dash. Drafting the article: Rathore, Dash. Critically revising the article: Rathore, Radhakrishnan. Reviewed submitted version of manuscript: Rathore. Approved the final version of the manuscript on behalf of all authors: Rathore. Statistical analysis: Sarma.

\section{Supplemental Information}

\section{Previous Presentations}

Portions of this work were presented in poster form at the 29th International Epilepsy Congress, Rome, Italy, August 28-September 1,2011, and published in the proceedings of the conference: Epilepsia 52(Suppl 6):23-263, 2011.

\section{Correspondence}

Chaturbhuj Rathore: Smt. B. K. Shah Medical Institute and Research Center, Gujarat, India. cbrathore@rediffmail.com. 